\title{
HISTORY OF A CASE
}

or

\section{HYDROMETRA}

\section{AND \\ DRY G A N G R E N E \\ OCCURRING}

IN THE SAME INDIVIDUAL,

WITH SOME OBSERVATIONS ON THESE DISEASES.

Bx ANTHONY TODD THOMSON, M.D. F.x.s.

SURGEON TO THE CHELSEA AND BROMPTON DISPENBARY, \&C.

Read June 14, 1825.

There is much truth in the remark that singular and extraordinary cases are seldom.instructive; but, if any practical inference can be drawn from them, they should, undoubtedly, be recorded; and, in this respect, I trust the details of the following case, in.which two diseases of rare occurrence appeared in the same individual, at the same time, will not be uninteresting to the society.

Mary Rae, aged sixty-five years, was admitted 
as a patient, into the infirmary of the Chelsea workhouse, under the care of Mr. Graskell, the Parochial Surgeon, on the first of April, 1823. She was a widow, her husband having died insane in 1819, up to which period she was of temperate and industrious habits, and enjoyed good health ; but, on the death of her husband, she became intemperate in the use of spirituous liquors, and a martyr to rheumatism. She had married early in life, and it is important to know that she had borne two children. The symptoms under which she laboured, at the time of her admission into the infirmary, were those of acute Rheumatism, affect ing chiefly the knees and ancles. The degree of fever was considerable; and, being a woman of sanguine temperament, she was confined to bed, freely bled and purged, and kept on low diet. She took as medicine, the Colchicum, both in substance and in its vinous preparation; and occasionally the oil of Turpentine : under which plan of treatment she was so much relieved, in two months, that she was discharged convalescent. She did not, however, recover her strength; and having probably returned to her former habits of intemperance, her health became again so much impaired, that five months afterwards, viz: early in December, 1823, she was re-admitted into the infirmary of the workhouse.

She appeared, now, somewhat emaciated; and complained of uneasiness and pain, connected with 
a tumour in the abdomen, which she informed Mr. Gaskell she had first perceived about six weeks prior to her admission into the infirmary in April, although, from a sense of delicacy, she had not mentioned it at that time. It was situate in the lower part of the abdominal cavity, rising, as it were, out of the pelvis, and occupying the iliac, hypogastric, and umbilical regions. She appeared as large as if six months gone with child. An indistinct fluctuation was perceptible in the tumor, and the least pressure on it excited pain. It was suspected to be a diseased ovarium; but no examination was made per vaginam; nor could it be ascertained, from the account which the patient gave of its origin, whether it had, at first, appeared on either side of the abdomen. The accompanying symptoms however, denoted a greater derangement of the system than usually attends Dropsy of the ovarium. These were, want of appetite, considerable nausea, furred tongue, the pulse quick and feeble, the bowels irregular, and the urine scanty and high coloured. It was deemed expedient, that she should be again confined to bed, and put on a vegetable diet. Mercurials were prescribed so as to affect the mouth slightly; the bowels were kept open; and leeches and blisters were alternately applied on the abdomen over the tumor. By these means its increase was arrested; but the sensation of pain, on pressure, remained unabated. 
Matters proceeded in this manner until the middle of January, 1824, when the left foot was discovered to be affected with dry Gangrene, which gradually extended nearly to the knee. I was now requested to see the patient. I found her in the state which has been described. The diseased foot and leg were much shrunk, and appeared more like parts of a mummy than of a living being : or, as if a dark brown, or blackish leather stocking were drawn over the limb of a skeleton; the whole being thin, dry, and harsh to the touch, and the natural divisions between the toes nearly obliterated. Although the state of the woman's health was not favourable for amputation, yet, as a feeble but distinct pulsation could be felt in the popliteal artery, and a line of separation between the diseased and the sound parts of the leg was fully formed, I concurred in opinion with $\mathrm{Mr}$. Gaskell that the removal of the limb afforded the only chance of preserving her life. The operation was delayed, however, in consequence of the reluctance of the patient to submit to it; but it was at length performed on the 29th of February, four inches above the knee. Very little blood was lost, and every thing appeared to be doing well until the third day, when the strength of the patient suddenly sunk, and she died on the evening of that day.

Before proceeding to detail the appearances on dissection, it may be proper to describe the state 


\section{DR. A. T. THOMSON'S CASE OF HYDRQMETRA}

of the amputated limb. Its external aspect has been described. On cutting through the integuments, the parts beneath, cellular matter, muscles, ligaments, and blood-vessels, seemed changed into one undistinguishable ash-coloured mass, not unlike the internal texture of some of the dry vegetable Fungi. In several parts, however, this was mixed with a semifluid matter. I endeavoured to trace the arteries, by injecting them from the sound part of the limb; but the line of separation was so complete as to render that attempt abortive.

\section{DISSECTION.}

The first object which presented itself, on the abdominal parietes being divided and turned aside, was a body closely resembling the gravid uterus, occupying the whole of the pelvic cavity, and the greater part of the abdominal. Upon its anterior surface, and firmly albering to it, was the urinary bladder, containing a small quantity of deep coloured urine. On laying the flaps of the abdominal parietes together, the stretched bladder was found to extend within an inch of the umbilicus; so that it must have been perforated, if the trochar had been employed to evacuate the fluid during the life of the patient, under the supposition that the disease was Ovarian-dropsy. The tumor was immediately ascertained to be the uterus, greatly enlarged and filled with fluid. It was partially sphacelated in its peritoneal covering, on the upper portion or fundus. 
With regard to the other viscera, the liver was much diminished in size, and adhered to the dia. phragm throughout: the gall-bladder was large, and turgid with deep coloured bile : the stomach, colon, and other intestines, with the omentum, were glued together in many places, and, in some, were in an evident state of sphacelation. This gangrenous appearance extended to the perito. neum, in the hypochondriac region. On removing the diseased uterus from the body, and making an incision into it, the quantity of fluid which it contained was found to measure eight quarts. It was of a dark brown colour, and coagulated slightly when heated in a spoon over the flame of a candle. The existence of a large hydatid within the cyst was suspected; but this opinion was incorrect, the sac being merely the uterus, in the cavity of which the fluid was contained. The internal surface of the organ was not more irregular nor more spongy than in its natural state, but none of the orifices could be found, for even the os uteri, was interiorly, as completely obliterated as if it had never existed; and although its situation could be traced in the vagina, yet even there it was very faintly marked. The ovaria were small and flaccid, but otherwise natural.

On examining the state of the larger blood-vessels within the abdomen, in reference to the diseased limb, the aorta was discovered to be ossified about two inches above its division into the iliac 
arteries. The right iliacs were in a healthy state; but the left external iliac was ossified in several places, in some of which the bony portion embraced nearly the cylinder of the vessel. . The ossific matter appeared to be deposited between the innermost and the second coats of the vessels. No traces of ossification were observed in the course of the femoral artery, and, as has been already stated, this vessel pulsated in the ham, although the lateral vessels, the muscular and articular arteries, which are given off from the popliteal artery, were shrunk and nearly obliterated.

It is probable that both the diseases under which this unfortunate woman laboured, originated from the same remote cause, the intemperate use of spirituous liquors. The examination of the body suggested a few physiological and pathological remarks, which I will now take the liberty of laying before the Society, in the hope that they may prove useful in a practical point of view.

In many of the cases of dry gangrene recorded by authors, the arteries leading to the diseased limb, besides being ossified, have been choked up with coagula*, which, impeding the supply of

- Vide Professor Avisard's cases in the Bibliothèque Medicale, for June, 1819. 
blood to the extreme vessels, readily explains the death of the limb : but, in other instances, as well as in that which I have described, no obstruction by coagula was discovered in the larger arteries above the diseased part, although, in all, there appears to have been ossification. From this fact, may it not be inferred, that the presence of the coagula was the consequence of the ossification; and that this diseased state alone, when extensive, is sufficient to induce dry Gangrene? A physiological question here suggests itself. How does the fact, that the ossification of the arterial trunks impedes the circulation so much in the extreme vessels, as to occasion the death of the limb, accord with the opinion, that the circulation is carried on altogether by the power of the heart, and that the arteries have no influence whatever in propelling the blood? If we admit the opinion that the arteries are passive tubes, we must, also, admit, that the propelling power of the heart should be sufficient to carry the blood forward to its extreme limit, even if the arteries were ossified through their whole extent? But, in the case before us, we find, that the simple ossification of a small portion of the aorta, and of several portions of the external iliac artery were sufficient, independent of the formation of coagula in these vessels, so to impede the supply of blood to the limb of the affected side, as to occasion its death. This is a subject, to the consideration of which, I must confess, my attention has not been much directed; but $I$ am disvoL. XIII. 
posed to think, that the state of the vessels, in the case before us, furnishes a strong argument against the accuracy of the hypothesis to which I have alluded.

It is a curious fact, that many of the recorded cases of dry Gangrene, besides that which is now under consideration, have occurred in gouty or rheumatic individuals* : and, even in some instances in which this is not particularly stated, a similar diathesis probably existed. Thus, in a case communicated by Doctor Alix, of Altenburg, and quoted by Doctor Mason Goodt, there was at first, pain and evident inflammation in the arm, the whole of which was afterwards affected with dry Gangrene, and a year after the appearance of that disease in the limb it separated spontaneously at the shoulder joint, leaving the patient, a man of seventy years of age, in good health. May we not infer from these cases, that the exciting cause of dry Gangrene is an inflammatory state of the vasa vasorum of the larger arteries, resembling that which occurs in the extreme arteries in gout and rheumatism, occasioning the deposition of osseous matter between their innermost and second coats; and, thence, diminishing or destroying that vis a tergo, the existence of which is necessary to be main-

* Vide Morgagni de Sed. et Causis Morb. Lib. iv. Epist. Iv. $\$ 24$, et seq.

+ See Study of Medicine, vol. ii. p. 919, first edit. and Observata Chirurgica, Fasciculus 1. 8vo. Altenburg, 1778. 
tained in these vessels for furnishing a due supply of blood to the extremities? Were the truth of this conjecture proved, it would certainly assist us in checking the progress of dry Gangrene, even after it has commenced in a limb, which has, hitherto, not been accomplished. But it is asserted, that the disease occurs when no inflammation exists. On this point I am sceptical; and would rather believe, that the inflammation may have been so obscure as to have escaped observation, than that it had not existed previous to the appearance of the gangrene.

Although the case before us terminated fatally soon after the amputation, yet I am not disposed to think, that the death of the patient was hastened by that operation, but by the morbid condition of the abdominal viscera. The operation was authorised by the pulsation in the popliteal artery, and the formation of a line of separation between the living and dead portions of the limb. These circumstances, indeed, are the only guides by which we can judge of the propriety of amputating in dry Gangrene, as they afford some reason for believing, that the yet living portion of the limb may be nourished and preserved. On the contrary, what benefit can be expected from that operation, when no line is formed, and no pulsation is perceptible in the larger vessels? In such a case, we may suspect that the principal artery which supplies the diseased limb is actually choked up, 
and the supply of blood, nearly, if not entirely, cut off; and by removing the dead portion, under such circumstances, we cannot expect to arrest the progress of decay in the part which is left.

The other disease of the individual whose case is now before the Society, is still of rarer occurrence than dry Gangrene; for, although several instances of water collected in the cavity of the uterus have been recorded, yet, very few of these can be regarded as cases of Hydrometra, in what may be termed its pure and unmixed state. Bonetus* gives the history of a case in which the uterus was enlarged to such an extent, that, to use the words of the author, "puer decem annorum facile potuerit in eo delitescere." The os uteri was closed by a round tumor, the size of a pomegranate, which was composed of a tenacious white concrete matter, resembling gluten. Hartmann tdescribes a case in which the mouth of the uterus was, also, stopped up by a swelling, the size of a goose's egg; and another, approximating in some degree to our case, in which he says, "orificium uteri in solidum ligamentum impervium degenerat" $\ddagger$; but the fluid, in this instance, was contained in two cells which did not communicate with one another.

\footnotetext{
* Anatomia Practica, lib. iii. sect. xxi ; obs. lv. $\oint 8$.

+ Miscellanea Cur. Acad. Naturæ Curiosorum, Dec. 2. Ann. 1686. Obs. 67.
}

$\ddagger$ Ibid Obs. 68 . 
In a case dissected by Monro*, the os uteri was swollen, knotty and scirrhous. Other cases are detailed in the collections of Haller, Borellus, and Lieutaud, Loder's Journal of Medicine, and the works of several other writers + : but the case which most resembles ours, although greatly exceeding it in the quantity of fluid contained in the uterus, which has fallen within the course of my reading, is one described by Vesalius $\ddagger$, in which the os uteri was firmly grown together, and the uterus contained one hundred and eighty pints of watery fluid.

Some modern writers, however, among whom is Doctor Denman, have doubted altogether the existence of pure Hydrometra. A case which came under the notice of that celebrated accoucheur, terminated by the spontaneous discharge of the water per vaginam, followed by a membranous bag, which, being inflated, assumed the figure of the distended uterus. He, therefore, concludes, "s that what has been called dropsy of the uterus, is probably no more than one large hydatid" $\zeta$. It cannot be denied, that a large hydatid may distend

* See his Collected Works, 4to.

t Those who are desirous of investigating this subject, may consult, for references, to recorded cases, Voigtel's Handbuch der Pathologischen Anatomie, vol. I1r. or Plouquet's Litteratura Medica.

$\ddagger$ De Corp. Hum. Fabrica. lib. v. cap. 9. p. 627.

$\S$ Introduction to Midwifery, vol. 1. p. 119. 
the uterus, and put on the character of pure $\mathrm{Hy}$ drometra : but, it is equally certain, that water may be accumulated in the cavity of the uterus, independent of the presence of any hydatid, as illustrated in the uterus now on the table of the Society.

The exciting cause of dropsy of the womb is not very obvious. Some authors refer it to whatever closes the os uteri, which, according to Astruc*, may be effected by any of the three following circumstances :

“ 1. Par obstruction, lorsque quelque matiére mucilageneuse qui coule de la matrice, s'y arrete, et s'y epaissit jusqu'a y demeurer collie.

“2. Par compression, lorsque l'orifice de la matrice est pressé par quelque tubercule squirrheux, ou par quelque callosité, qui s'y est formée à la suite de quelque accouchement laborieux.

“ g. Par constriction, lorsque cet orifice est fortement resserri par quelque contraction convulsive, comme il arrive souvent dans la passion hysterique."

He might have added, as a fourth cause, by such a degree of inflammation as completely obliterates the os uteri. Hartmann, speaking of the case he

* Des Maladies des Femmes, liv. 2. p. 337. 
describes, also remarks,-“" Hydropis uteri prima causa in hac, constrictio orificii aut colli uteri"* : and it is very evident that no accumulation of fluid can happen in this organ, unless the os uteri be either entirely obliterated, as in the case before us, or otherwise obstructed. These conditions of the orifice of the organ, however, merely explain the mechanical means by which the fluid is retained in the distended uterus, but throw no light on the causes of its production.

In the unimpregnated state of the uterus, the internal surface or cavity is necessarily kept moist by an exhaled fluid; but, as we have no observations which prove that this fluid escapes into the vagina, we must conclude that, like the exhalations into the other cavities of the body, it is taken up and carried away by the absorbents. A due balance of the action of the exhalants and the absorbents is necessary, therefore, for maintaining that quantity of this fluid which is required for the purposes of the organ; and we may reasonably suppose, that this proportion would be preserved, were the orifice closed by any means whatsoever, provided the health of the uterus itself remained unaffected. Some authors have therefore sought to ascribe the collection of the fluid to other causes. Thus Carron, quoted by Dr. Goodt, ascribes it, in

* Loco citato. † Study of Medicine, vol. Iv. 
various cases, to a debility of the uterus produced by a chronic leucorrhæa. Others have imagined, that it may arise from the stimulus of pentup coagulated blood; and Doctor Baillie, without adverting to the state of the organ, thinks it is probable, " that the fluid resembles in its properties the serum, and is poured out by the small curling arteries of the uterus"*.

Were debility of the uterus the cause of the accumulation of water in its cavity, admitting that the quantity poured out by the exhalants is suffciently great to produce the distention which occurs, and that the absorbents are paralyzed, or otherwise rendered incapable of taking it up, we should expect to find the sac thin in proportion to its size. It is evident, however, from the appearance of the paries of the enlarged uterus before us, that it was not mere distention, by the pressure of the fluid which it contained, that enlarged it; but, that the increase of bulk has arisen from some new action in the vessels of the part. Is it more difficult to suppose, that the increments of growth in the uterus, in its dropsical state, results from the diseased action which causes the preternatural increase of its fluid, than in a similar morbid condition of the ovarium, which, from being in its healthy state, an insignificant body in point of size, becomes a strong, thick, membranous sac, 
capable of containing several gallons of water? Judging from the manner in which the os uteri is closed, in the case before us, it is probable, that in some instances, the new action is of an inflammatory kind, and if we may support our opinion by analogy, we know that water is effused into the ventricles of the brain, and other cavities supplied with a moist exhalation, from the same cause. With respect to the opinion that the disease may proceed from the stimulus of pent-up coagulated blood, we need only remark, that moles and other collections of blood are occasionally found in the uterus, without the presence of any dropsical effusion.

The greater frequency of ovarian dropsy, and the difficulty of the diagnosis, without an examination per vaginam, may have, occasionally, led prac. titioners to mistake uterine dropsy for that disease; and, in some of the instances of recorded dropsy of the ovarium, which were relieved by a spontaneous discharge of the fluid by the vagina, I am inclined to think that the water was contained in the cavity of the enlarged uterus. Such was probably the case in the curious instance mentioned by Johannes Benedictus Grundelius, which occurred in the person of a Hungarian Countess, in 1698, who supposed herself pregnant, as the menstrual discharge was suspended, and a tumor appeared in the abdomen, which gradually increased in size. In time, however, no movement of a child being 
felt, and falling into bad health, she was induced to use warm baths, when, having pursued this course for eight days, the orifice of the uterus was opened, and, adds the narrator, " magna humoris fotidi suburra unà cum copiosis flatibus ex utero evacuato" : after which she perfectly recovered, and was, subsequently, the mother of two children. In a case which came under my own observation, an unmarried woman of libertine habits, supposed that she was pregnant, and gradually increased in size during eleven months. No motion of a child was however perceptible; there was an evident fluctuation of the tumor when she applied for my advice, and believing that it was avarian dropsy, I proposed to evacuate the fluid by the trocar. She would not, however, consent to the operation, and a short time afterwards was suddenly relieved by a discharge of fluid per vaginam. She has since continued in good health. A similar case occurred, I am informed, in the practice of the uncle of the present Doctor Merriman : and in a letter which I received from my learned and excellent friend, Doctor John Thomson, of Edinburgh, is the following paragraph. "I have myself seen two cases, and I have heard of more from my acquaintance, in which great quantities of water have been periodically discharged from the uterus during many months : but, whether the water was formed in that viscus, or came from the ovarium, I have not been able to ascertain. In one of the cases to which I allude, the patient is 
still alive; and I have ascertained both by examination per vaginam, and by the rectum, that she has a disease of the neck of the uterus." These spontaneous evacuations of fluid, suggested the plan of curing the disease by paracentesis, but few cases are on record, in which that operation was actually performed.

In conclusion, we may perhaps be permitted to suggest the following corollaries:

1. That in incipient cases of dry Gangrene, when the exciting causes are not obvious, benefit may be derived from treating them in the same manner as cases of gouty or rheumatic inflammation; in addition to the local stimulants usually employed: i. e. with Calomel, Tartar-emetic, Opium, Colchicum, and Peruvian bark.

2. That pure Hydrometra, or an accumulation of a serous fluid in the cavity of the uterus, en. larged, and acquiring the character of a membranous sac, is a disease which occasionally occurs.

3. That in no case of supposed ovarian dropsy, should a trocar be passed into the tumor, through the linea alba, without ascertaining by previous examination per vaginam the real nature of the tumor. For, as in the case before the Society, should the uterus be the seat of the disease, and the bladder of urine be attached to its anterior surface, 
and drawn up nearly to the umbilicus, the instrument would pass through that viscus, and might produce irreparable mischief.

4. That the number of instances in which the disease has been cured by a spontaneous discharge of the fluid per vaginam, authorize the attempt to evacuate it artificially, either by dilating the os uteri, when it is only obstructed, or puncturing the sac, in the situation of that orifice, when it is obliterated. 